\title{
MicroRNA-361-5p inhibits epithelial-to-mesenchymal transition of glioma cells through targeting Twist1
}

\author{
XI ZHANG $^{1 *}$, CHUNYAN WEI $^{2 *}$, JIN LI $^{1}$, JIALI LIU $^{3}$ and JIANQIANG QU ${ }^{1}$ \\ Departments of ${ }^{1}$ Neurosurgery, ${ }^{2}$ Gynaecology and Obstetrics, and ${ }^{3}$ Clinical Laboratory, \\ The Second Affiliated Hospital of Xi'an Jiaotong University, Xi'an, Shaanxi 710004, P.R. China
}

Received July 21, 2016; Accepted September 9, 2016

DOI: 10.3892/or.2017.5406

\begin{abstract}
MicroRNA-361-5p (miR-361-5p) has been reported to be dysregulated in various human cancer types. However, the function of miR-361-5p in glioma remains unknown. In the present study, we aimed to investigate the biological functions of miR-361-5p in regulating glioma progression and the underlying molecular mechanism. We found that miR-361-5p was significantly decreased in glioma tissues and cell lines as detected by reverse transcription-quantitative polymerase chain reaction (RT-qPCR) analysis. Functional analysis revealed that miR-361-5p overexpression significantly inhibited glioma cell migration, invasion and epithelial-mesenchymal transition (EMT) whereas suppression of miR-361-5p showed opposite effects. Bioinformatic analysis showed that Twist1, a critical EMT inducer, was a predicted target of miR-361-5p which was validated by dual-luciferase reporter assay, RT-qPCR and western blot analysis. Further analysis indicated that miR-361-5p regulates the Twist1/Bmi-1 signaling axis. Rescue experiments showed that restoration of Twist1 expression significantly reversed the suppressive effect of miR-361-5p on cell migration, invasion and EMT. Taken together, the present study demonstrated an important role of miR-361-5p in glioma - which regulated the EMT of glioma cells by targeting and regulating Twist1. These findings provide novel insight into understanding the role and mechanism of miR-361-5p in regulating the biological behavior of glioma cells and suggest that miR-361-5p is a novel potential therapeutic target for glioma.
\end{abstract}

Correspondence to: Dr Jianqiang Qu, Department of Neurosurgery, The Second Affiliated Hospital of Xi'an Jiaotong University, No. 157 West 5th Road, Xi'an, Shaanxi 710004, P.R. China

E-mail: qujianqiangxa@163.com

*Contributed equally

Abbreviations: miRNAs, microRNAs; EMT, epithelial-mesenchymal transition; RT-qPCR, reverse transcription-quantitative polymerase chain reaction; 3'-UTR, 3'-untranslated region; WHO, World Health Organization; FBS, fetal bovine serum

Key words: glioma, miR-361-5p, Twist1, epithelial-mesenchymal transition

\section{Introduction}

Glioma represents one of the most common brain tumors causing severe mortality every year worldwide $(1,2)$. Due to the rapid growth, angiogenesis and metastasis of glioma cells, it is extremely difficult to cure this disease (3). Despite multiple advanced treatments over the past few decades, glioma remains an intractable disease with a poor prognosis $(2,4)$. Therefore, the urgent development of new therapies for glioma is essential. However, the molecular heterogeneity and the unclear pathogenesis of glioma still hamper the development of new treatments for glioma.

In recent years, microRNAs (miRNAs) have drawn considerable attention as potential and promising tools for cancer diagnosis and treatments $(5,6)$. miRNAs can regulate target gene expression by interacting with the 3'-untranslated region (3'-UTR) of mRNAs to inhibit translation or induce mRNA degradation $(7,8)$. Therefore, miRNAs are able to modulate cancer cell proliferation, apoptosis, differentiation, migration and invasion by targeting oncogenes or tumor suppressors (9). A growing body of evidence suggests that various miRNAs which are aberrantly expressed in glioma participate in glioma initiation and progression as well as chemoresistance and radioresistance $(10,11)$, highlighting their potential as novel agents for glioma therapies. However, their regulatory functions in glioma remain largely unknown.

Twist1 is a basic-helix-loop-helix family transcription factor and plays an important role in the development of many pathological diseases (12). A prominent function of Twist1 is the regulation of tumor metastasis by affecting epithelial-mesenchymal transition (EMT) (13). EMT is an essential process for cancer invasion and metastasis (14). Twist1 activation is sufficient to promote EMT and the dissemination of tumor cells, and Twist1 overexpression is associated with more invasive cancer types and a poor prognosis $(15,16)$. The hypoxic condition can induce the EMT process of tumor cells through activation of Twistl (17). Overexpression of Twist1 is frequently observed in a variety of types of cancers including hepatocellular carcinoma, prostate, breast, gastric and lung cancer, and plays an important role in regulating cancer initiation, progression and metastasis involving multiple regulatory pathways (12). High expression of Twist1 has been detected in a significant proportion of human gliomaderived cell lines and human glioma tissues (18) and was 
found to predict poor survival for glioma patients (19). Twist1 overexpression was also found to promote cell invasion while suppression of Twist1 was found to inhibit the cell invasion of glioma cells $(18,20)$. Therefore, targeting Twist1 may have promising and potential therapeutic value as a glioma treatment.

Emerging evidence indicates that microRNA-361-5p (miR-361-5p) functions as a tumor suppressor in several types of cancers, such as hepatocellular carcinoma (21), prostate (22), gastric and colorectal cancer (23). However, the functional significance of miR-361-5p in glioma has not been investigated. In the present study, we aimed to investigate the biological functions of $\mathrm{miR}-361-5 \mathrm{p}$ in regulating glioma progression and the underlying molecular mechanism. We found that miR-361-5p expression was significantly lower in glioma tissues and cell lines compared to that obwerved in normal brain tissues. Overexpression of miR-361-5p inhibited glioma cell migration, invasion and EMT. In addition, bioinformatic analysis indicated that Twistl was a direct target of miR-361-5p in glioma cells. Our data suggest that miR-361-5p regulates glioma cell EMT through targeting Twist1. Our findings provide a better understanding of the mechanism of glioma development and suggest that miR-361-5p may serve as an attractive molecular target for the treatment of glioma.

\section{Materials and methods}

Tissue specimens. A total of 30 glioma tissue samples were obtained from the Department of Neurosurgery, The Second Affiliated Hospital of Xi'an Jiaotong University (Xi'an, China). The specimens were collected during tumor resection surgery prior to any chemotherapy or radiotherapies. The resected tumor samples were immediately snap-frozen in liquid nitrogen and stored at $-80^{\circ} \mathrm{C}$. Glioma tissue samples were classified according to the World Health Organization (WHO) standards. Gliomas (12 of the 30) were classified as low-grade (5 WHO I and 7 WHO II) and 18 were classified as high-grade (8 WHO III and $10 \mathrm{WHO}$ IV). Ten samples of normal brain tissues were obtained from internal decompression patients undergoing surgical operation at The Second Affiliated Hospital of Xi'an Jiaotong University. Written informed consent was obtained from all patients. The present study was approved by the Institutional Human Experiment and Ethics Committee of The Second Affiliated Hospital of Xi'an Jiaotong University and was performed in accordance with the Helsinki Declaration.

Cell cultures. Human glioma cell lines U87, U251, SHG44 and A172, and normal human astrocytes were purchased from the Type Culture Collection of the Chinese Academy of Sciences (Shanghai, China). All cells were routinely cultured in Dulbecco's modified Eagle's medium (DMEM) plus 10\% fetal bovine serum (FBS) (both from Gibco, Rockville, MD, USA) and $1 \%$ penicillin/streptomycin mixture (Sigma, St. Louis, MO, USA) at $37^{\circ} \mathrm{C}$ in a humidified incubator containing $5 \% \mathrm{CO}_{2}$.

Reverse transcription-quantitative polymerase chain reaction $(R T-q P C R)$. Total RNA was extracted using TRIzol reagent (Invitrogen, Carlsbad, CA, USA). For detection of miR-361-5p, complementary DNA was synthesized by TaqMan MicroRNA
Reverse Transcription kit (Applied Biosystems, Carlsbad, CA, USA). To detect Twist1, cDNA was synthesized by M-MLV reverse transcriptase (Takara, Dalian, China) as per the manufacturer's instructions. PCR amplification was performed by SYBR-Green Master Mix kit (Bio-Rad, Hercules, CA, USA) on an Applied Biosystems AB7500 Real-Time PCR system (Applied Biosystems) with specific primers: miR-361-5p forward, 5'-ATAAAGTGCTGACAGTGCAGATAGTG-3' and reverse, 5'-TCAAGTACCCACAGTGCGGT-3'; U6 forward, 5'-CGCTTCGGCAGCACATATACTAA-3' and reverse, 5'-TA TGGAACGCTTCACGAATTTGC-3'; Twist1 forward, 5'-CC AGGTACATCGACTTCCTCTA-3' and reverse, 5'-CCATCCT CCAGACCGAGAA-3'; and GAPDH forward, 5'-CCATGTT CGTCATGGGTGTG-3' and reverse, 5'-GGTGCTAAGCAGT TGGTGGTG-3'. U6 and GAPDH were used as internal normalized references. Relative miRNA or mRNA expression was calculated using the $2^{-\Delta \Delta C t}$ method, normalized against U6 or GAPDH and then compared with the control group.

Transfection of miRNA. miR-361-5p mimics, a miR-361-5p inhibitor and a scrambled miRNA control were purchased from GenePharma (Shanghai, China) and transfected into cells using Lipofectamine 2000 (Invitrogen) at a final concentration of $50 \mathrm{nM}$ according to the manufacturer's instructions. After $48 \mathrm{~h}$ of transfection, the transfection efficacy was determined by RT-qPCR analysis.

Migration and invasion assays. For detection of cell migration, $1 \times 10^{5}$ cells transfected with miR-361-5p mimics or miR-361 inhibitor were resuspended in $500 \mu \mathrm{l}$ of medium without FBS and placed in the upper chambers of the Transwell (Costar, Cambridge, MA, USA). The lower chambers were filled with medium containing $10 \%$ FBS as a chemoattractant. For detection of cell invasion, the upper chambers were coated with Matrigel (BD Biosciences, San Jose, CA, USA). After $24 \mathrm{~h}$ at $37^{\circ} \mathrm{C}$, the non-migrating or non-invading cells on the top well were gently scraped. The cells on the lower surface of the membrane were fixed with $70 \%$ ethanol and stained with $0.1 \%$ violet (Sigma). The cells were observed and counted under a microscope (Olympus, Tokyo, Japan).

Western blot analysis. Cells were lysed with a cell lysis buffer and the protein concentration was detected using a BCA protein kit (Beyotime, Haimen, China). A total of $50 \mu \mathrm{g}$ of protein was separated by SDS-PAGE and electro-transferred to a polyvinylidene fluoride (PVDF) membrane (Bio-Rad). The membrane was then blocked using 5\% non-fat milk and incubated in the primary antibody (anti-E-cadherin, anti-N-cadherin, anti-vimentin, anti-Twist1, anti-Bmi-1 and anti-GAPDH; purchased from Santa Cruz Biotechnology, Santa Cruz, CA, USA) at $4^{\circ} \mathrm{C}$ overnight. Thereafter, the membrane was washed and incubated with horseradish peroxidase-conjugated secondary antibodies (Bosis, Beijing, China) for $1 \mathrm{~h}$ at $37^{\circ} \mathrm{C}$. The protein bands were visualized using an ECL Western blotting kit (Pierce, Rockford, IL, USA) according to the manufacturer's instructions. Densitometric analysis of the protein bands was performed using Image-Pro Plus 6.0 software (Media Cybernetics, Inc., Rockville, MD, USA). The values were normalized against GAPDH and were then compared to the control group. 

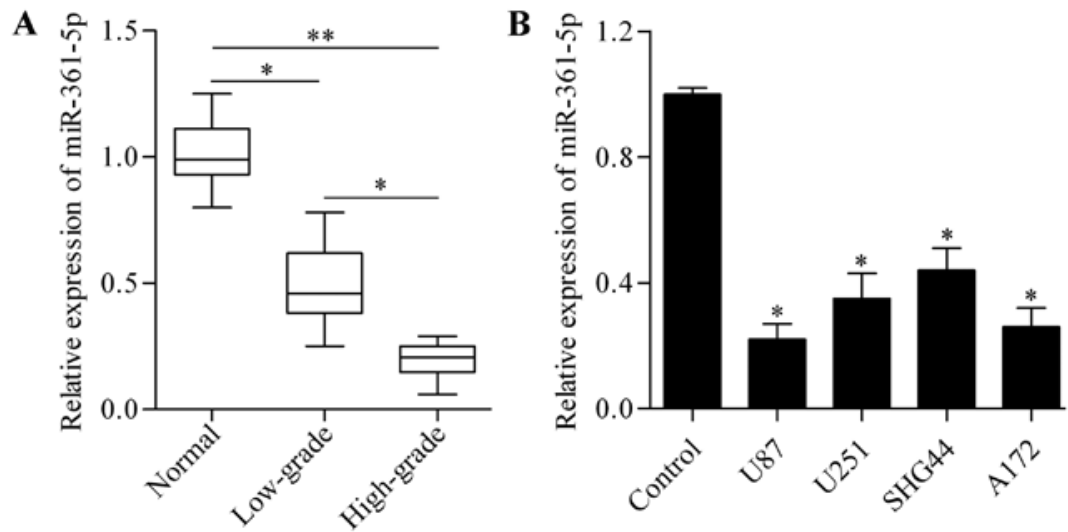

Figure 1. miR-361-5p is downregulated in glioma. (A) RT-qPCR analysis of miR-361-5p in low-grade glioma, high-grade glioma and normal brain tissues; ${ }^{*} \mathrm{p}<0.05,{ }^{* *} \mathrm{p}<0.01$. (B) RT-qPCR analysis of miR-361-5p in glioma cell lines (U87, U251, SHG44 and A172) and normal human astrocytes. Normal human astrocytes was used as the control; ${ }^{*} \mathrm{p}<0.05$ vs. the control.

Dual-luciferase reporter assay. A Twist1 cDNA fragment harboring seed-matched sequences with or without a mutation was inserted into the pmirGLO Dual-Luciferase vector (Promega, Madison, WI, USA). The pmirGLO vector was then co-transfected with miR-361-5p mimics or miR-361 inhibitor into glioma cells by Lipofectamine 2000 and incubated for $48 \mathrm{~h}$. Then, the relative luciferase activity was analyzed by a Dual-Luciferase reporter assay system kit (Promega).

Rescue assay. Full length Twist1 cDNA without the 3'-UTR region was cloned into the pcDNA3.0 vector (Invitrogen). The pcDNA/Twist1 vector was co-transfected with miR-361-5p mimics into glioma cells using Lipofectamine 2000. After $48 \mathrm{~h}$, TWIST1 expression was detected by RT-qPCR and western blot analysis.

Statistical analysis. Data are expressed as means \pm standard deviation. Statistical analyses were performed using SPSS version 11.5 (SPSS, Inc., Chicago, IL, USA). Differences were calculated by Student's t-test or one-way analysis of variance followed by Bonferroni post hoc test. At a p-value $<0.05$, the difference was considered to be statistically significant.

\section{Results}

miR-361-5p is reduced in human glioma tissues and cell lines. To investigate the potential biological role of miR-361-5p in glioma, we first detected the expression of miR-361-5p in glioma tissues and cell lines by RT-qPCR analysis. The results showed that the expression of miR-361-5p was significantly downregulated in glioma tissues and miR-361-5p expression was significantly lower in high-grade than low-grade glioma tissues (Fig. 1A). miR-361-5p was also markedly lower in the U87, U251, SHG44 and A172 glioma cell lines as compared with the level in the normal human astrocytes (Fig. 1B). These results indicate that miR-361-5p may play an important role in glioma.

miR-361-5p inhibits cell migration and invasion of glioma cells. To investigate the biological effect of miR-361-5p in glioma cells, we performed gain-of-function and loss-of-function experiments by transiently transfecting miR-361-5p mimics or
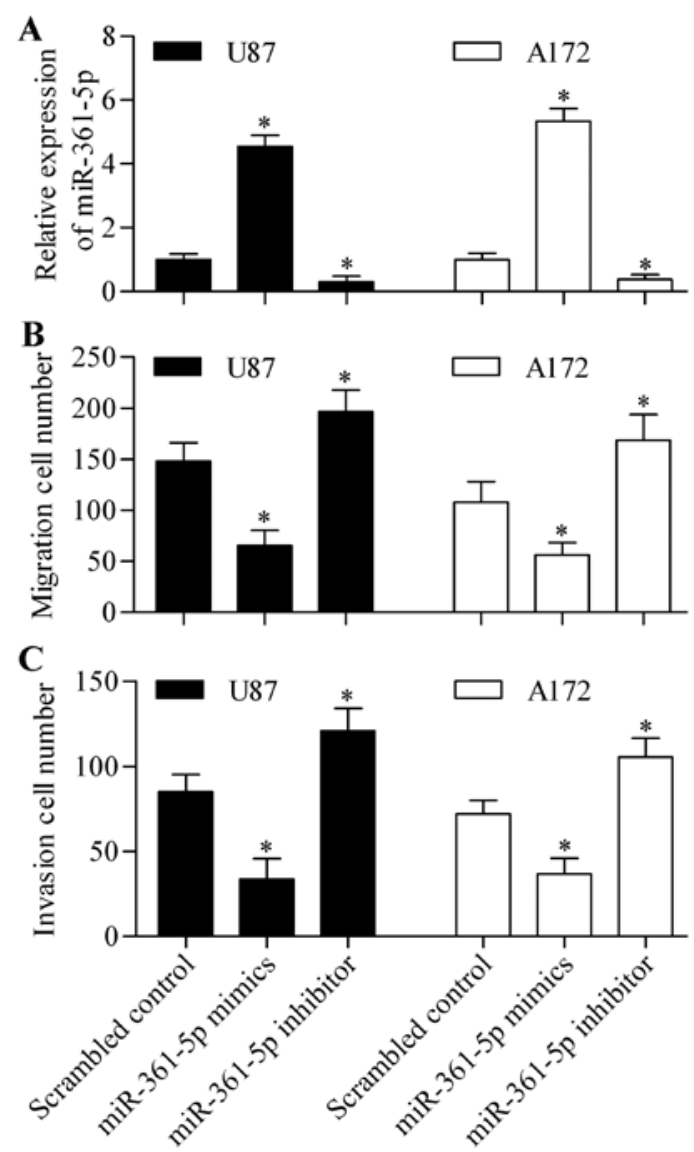

Figure 2. miR-361-5p inhibits cell migration and invasion of glioma cells. (A) RT-qPCR analysis of miR-361-5p expression in U87 and A172 cells transfected with miR-361-5p mimics or miR-361-5p inhibitors for $48 \mathrm{~h}$. The effect of miR-361-5p overexpression or miR-361-5p suppression on the (B) migration and (C) invasion ability of the glioma cells was detected by Transwell assay; ${ }^{*} \mathrm{p}<0.05$ vs. the scrambled control.

miR-361-5p inhibitors into U87 and A172 cells. Transfection of the miR-361-5p mimics significantly increased miR-361-5p expression whereas transfection of the miR-361-5p inhibitors significantly suppressed miR-361-5p expression (Fig. 2A). We then examined the role of miR-361-5p in glioma cell migration and invasion. We determined that overexpression of miR-361-5p significantly decreased migration (Fig. 2B) 
A

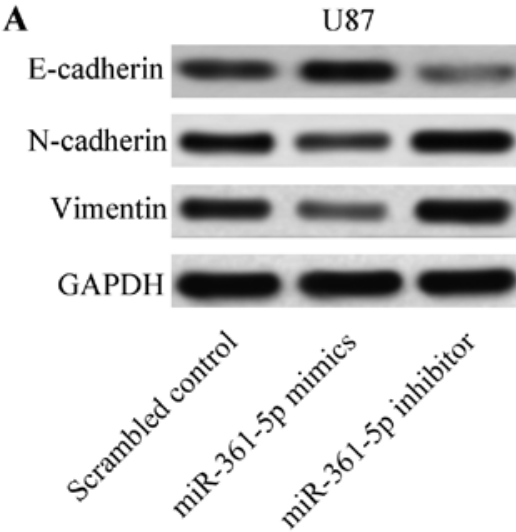

C

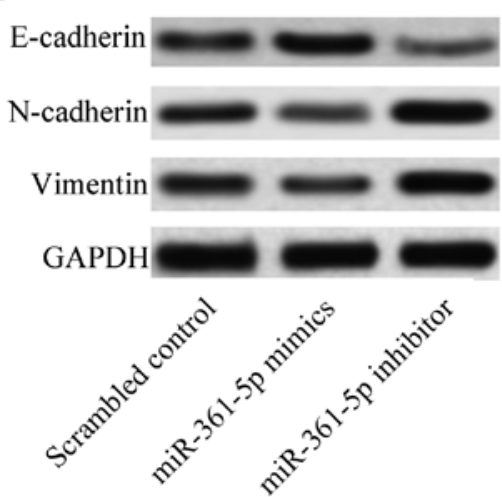

B

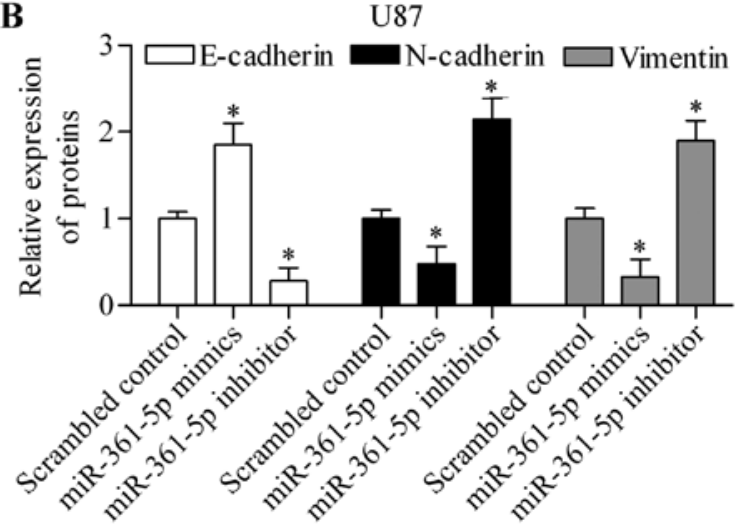

D

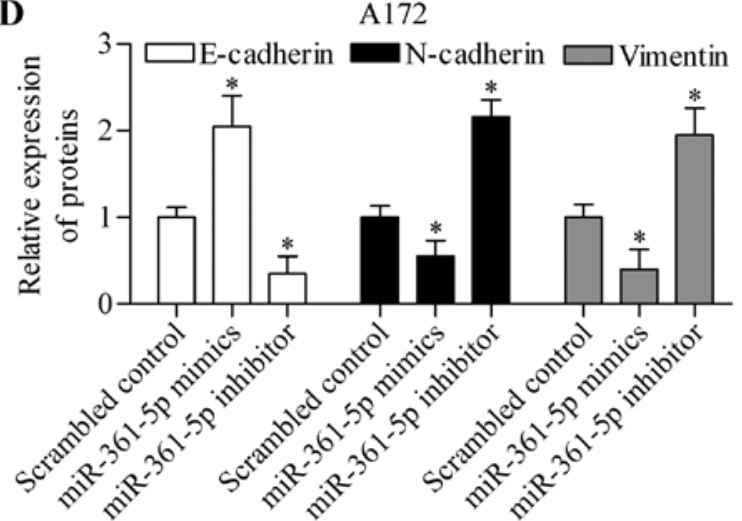

Figure 3. miR-361-5p inhibits epithelial-mesenchymal transition (EMT) in glioma cells. Western blot analysis of E-cadherin, N-cadherin and vimentin in (A) U87 and (C) A172 cells. The relative expression of proteins in (B) U87 and (D) A172 cells was quantified using Image-Pro Plus 6.0 software; * $<0.05$ vs. the scrambled control.

A

$$
\begin{aligned}
& \text { 3'-caUGGGGACCUCUAAGACUAUu-5' } \\
& \text { | | | | | } \\
& \text { 5'-acACCUCUG----CAUUCUGAUAg-3', } \\
& \text { 5'-acACCUCUG----CUGGAACUCGg-3' }
\end{aligned}
$$

Hsa-miR-361-5p

Twistl 3'-UTR (WT)

Twist1 3'-UTR (MT)

$\mathbf{B}$

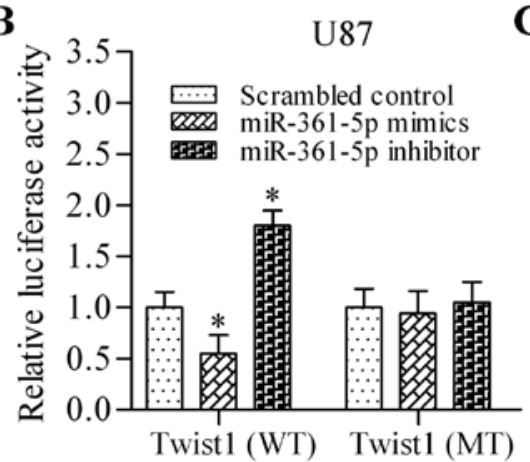

C

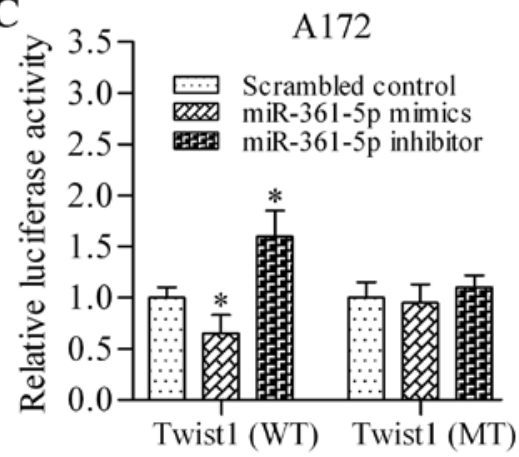

Figure 4. miR-361-5p targets the 3'-UTR of Twist1. (A) Schematic representation of the seed region between miR-361-5p and the 3'-UTR of Twist1. The luciferase activity of (B) U87 and (C) A172 cells was detected after co-transfection with the miR-361-5p mimics or miR-361-5p inhibitors and wild-type (WT) or (MT) mutant-type report vectors by a dual-luciferase reporter system; ${ }^{\mathrm{p}}<0.05$ vs. the scrambled control.

and invasion (Fig. 2C). In contrast, miR-361-5p suppression markedly promoted cell migration (Fig. 2B) and invasion (Fig. 2C). These data suggest that miR-361-5p functions as a suppressor of cancer cell metastasis.
miR-361-5p inhibits EMT of glioma cells. To further investigate the biological effect of miR-361-5p on glioma, we investigated the role of miR-361-5p in glioma cell EMT. We determined that miR-361-5p overexpression significantly increased the 
A

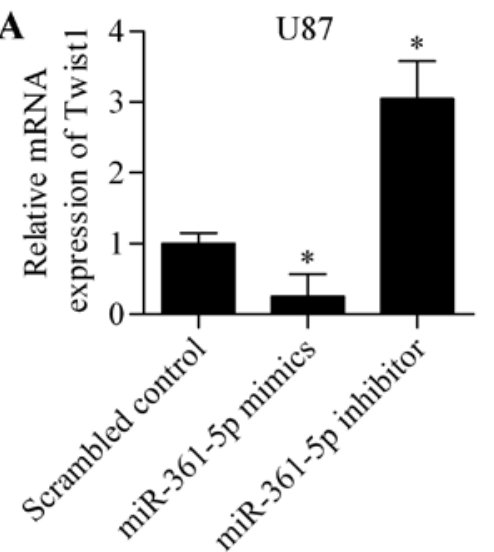

C

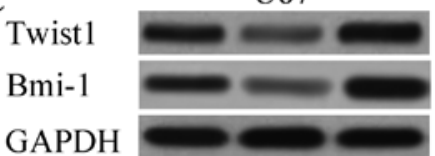

E

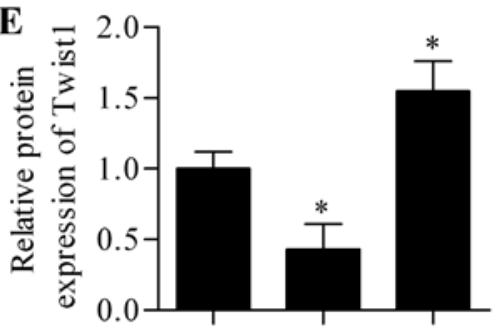

G

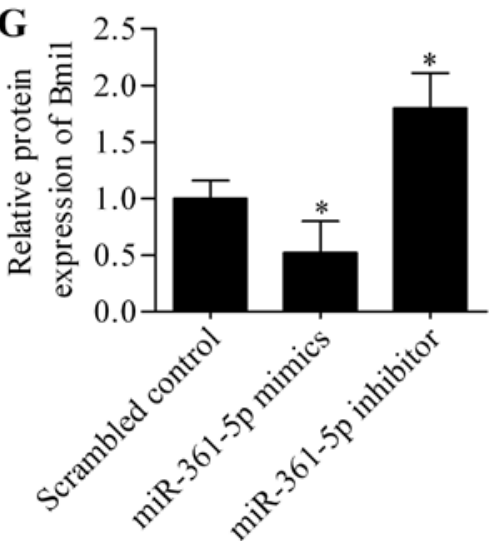

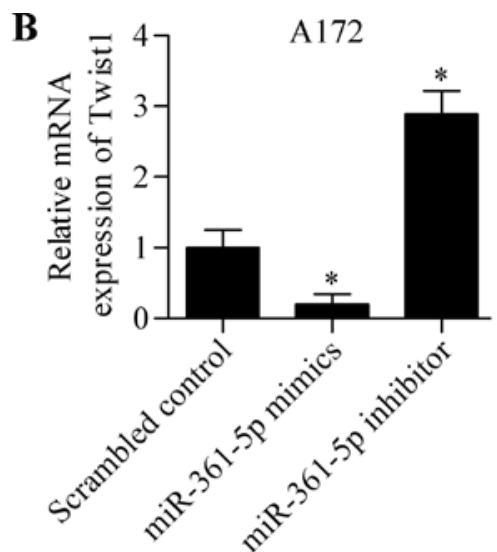

D
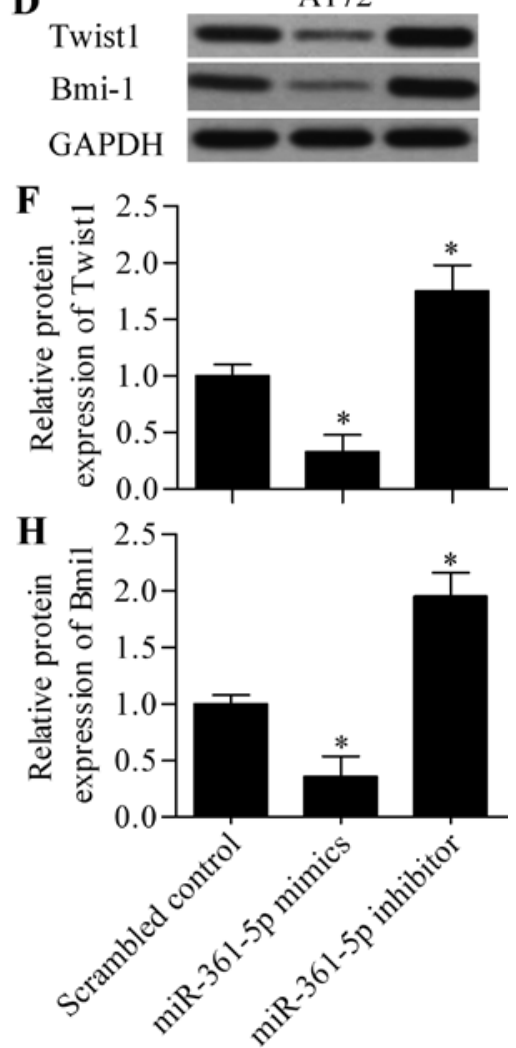

Figure 5. miR-361-5p inhibits Twist1 expression. U87 and A172 cells were transfected with miR-361-5p mimics or miR-361-5p inhibitors for 48 h. The mRNA expression of Twist1 in (A) U87 and (B) A172 cells was detected by RT-qPCR analysis. The protein expression of Twist1 and Bmi-1 in (C) U87 and (D) A172 cells was detected by western blot analysis. The relative protein expression of (E and F) Twist1 and (G and H) Bmi-1 in U87 and A172 cells was quantified using Image-Pro Plus 6.0 software; " $\mathrm{p}<0.05$ vs. the scrambled control.

expression of epithelial marker E-cadherin and inhibited expression of mesenchymal markers $\mathrm{N}$-cadherin and vimentin in the U87 (Fig. 3A and B) and A172 cells (Fig. 3C and D). Conversely, miR-361-5p suppression exhibited an opposite effect (Fig. 3A-D). These results suggest that miR-361-5p suppresses EMT of glioma cells.

Twist1 is a target of miR-361-5p in glioma cells. To understand the molecular basis of miR-361-5p in regulating glioma cell EMT, we conducted bioinformatic analyses to identify the target of miR-361-5p in glioma. Our analysis revealed Twist1 as a potential target gene of miR-361-5p. The 3'-UTR of Twistl was found to contain a binding sequence for miR-361-5p (Fig. 4A). To confirm that Twistl is a target gene of miR-361-5p, a dual-luciferase reporter assay was performed. The results showed that miR-361-5p overexpression significantly inhibited the luciferase activity in the U87 (Fig. 4B) and A172 (Fig. 4C) cells transfected with the luciferase reporter vector containing the wild-type (WT) Twist1 3'-UTR whereas miR-361-5p suppression promoted the luciferase activity. However, neither miR-361-5p overexpression nor miR-361-5p suppression showed an obvious effect on luciferase activity of the mutant-type (MT) Twist1 3'-UTR. Moreover, we next detected the direct effect of miR-361-5p overexpression or miR-361-5p suppression on Twist1 expression in glioma cells by RT-qPCR and western blot analysis. The results showed that overexpression of miR-361-5p significantly inhibited both the mRNA (Fig. 5A and B) and protein (Fig. 5C-F) expression 

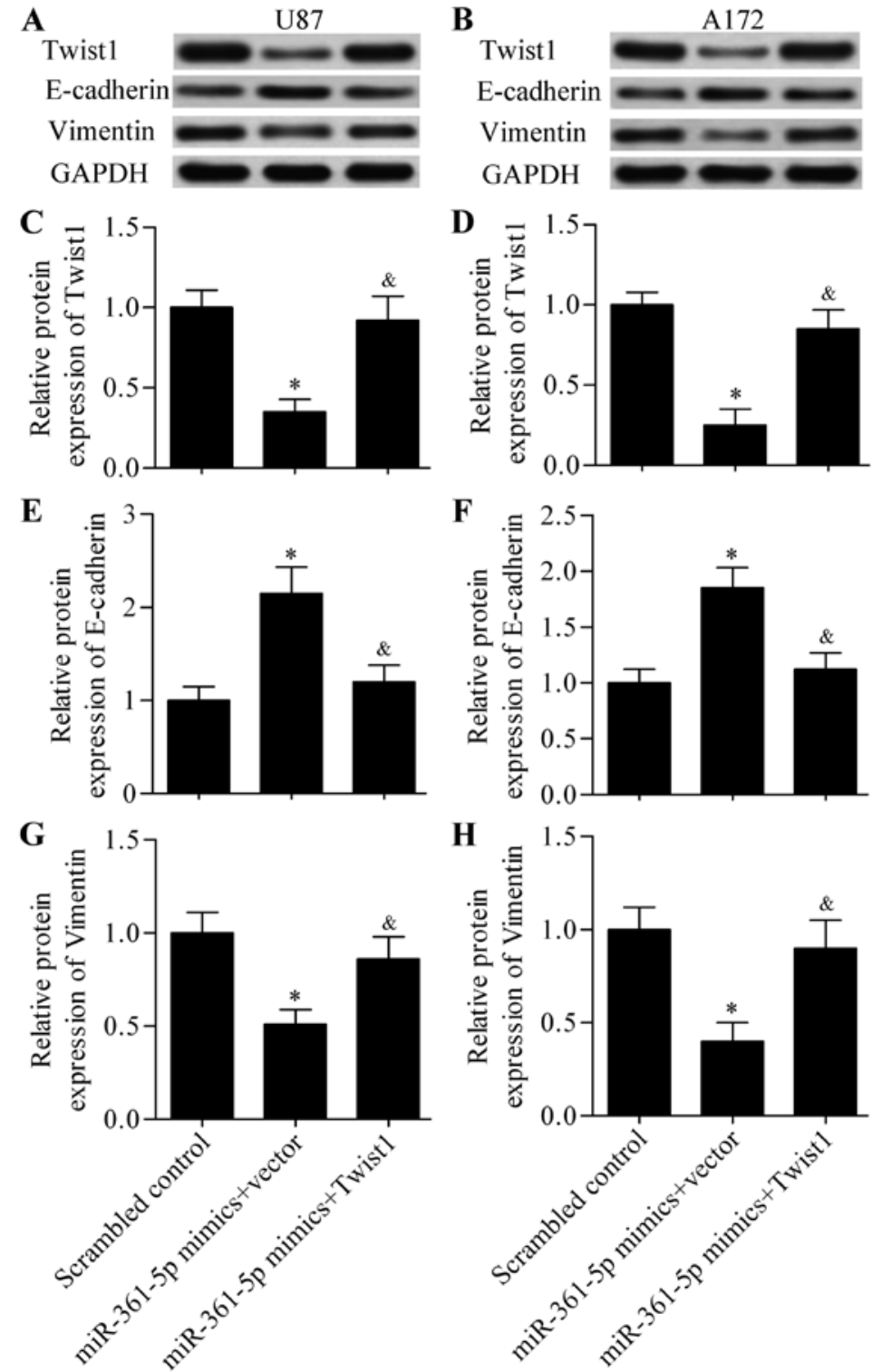

Figure 6. Overexpression of Twist1 rescues the inhibitory effect of miR-361-5p on epithelial-mesenchymal transition (EMT). Cells were co-transfected with the miR-361-5p mimics and pcDNA/Twist1 vector without 3'-UTR. The protein expression level of Twist1, E-cadherin and vimentin in (A) U87 and (B) A172 cells was detected by western blot analysis. The relative protein expression of (C and D) Twist1, (E and F) E-cadherin and (G and H) vimentin in U87 and A172 cells was quantified using Image-Pro Plus 6.0 software; ${ }^{*} \mathrm{p}<0.05$ vs. the scrambled control; ${ }^{\&} \mathrm{p}<0.05$ vs. the miR-361-5p mimics + vector.

levels of Twist1 which were markedly increased by miR-361-5p suppression. These results suggest that miR-361-5p targets the 3'-UTR of Twist1 and inhibits Twistl expression.

miR-361-5p regulates Bmi-1 expression. Bmi-1 is a well-known downstream gene of Twist1 that participates in tumorigenesis and metastasis (24). We thus, investigated whether miR-361-5p regulates Bim-1. We found that overexpression of miR-361-5p significantly inhibited Bmil expression and miR-361-5p suppression significantly increased Bmi-1 expression (Fig. 5C and $\mathrm{D}$, and $\mathrm{G}$ and $\mathrm{H}$ ). The results suggest that miR-361-5p regulates the Twist1/Bmi-1 signaling axis.

Overexpression of Twist1 rescues the effects of miR-361-5p in glioma cells. To verify the functional relevance of Twist1 targeting by miR-361-5p, we performed a rescue assay to assess whether Twist1 overexpression rescues the inhibitory effects of
miR-361-5p. Glioma cells were co-transfected with miR-361-5p mimics and the Twist1-overexpressing plasmid. We determined that the reduced protein expression induced by miR-361-5p overexpression was significantly restored by transfection with the Twistl-overexpressing plasmid in the U87 (Fig. 6A and C) and A172 (Fig. 6B and D) glioma cells. Overexpression of Twist1 rescued the inhibitory effect of miR-361-5p on glioma cell EMT (Fig. 6A, B and E-H). Furthermore, the inhibitory effects of miR-361-5p on glioma cell migration (Fig. 7A) and invasion (Fig. 7B) were significantly reversed by Twist1 overexpression. These results suggest that miR-361-5p regulates EMT through repressing Twist1.

\section{Discussion}

A growing body of evidence suggests that miRNAs play a pivotal role in regulating the tumorigenesis and metastasis 

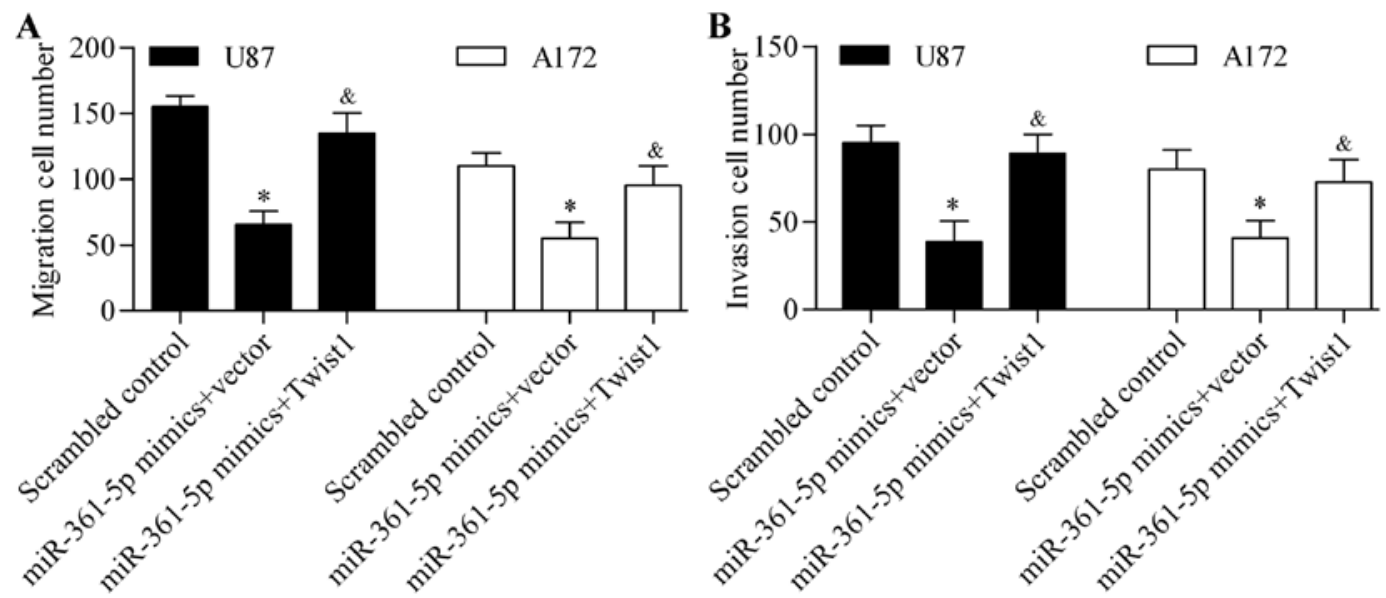

Figure 7. Overexpression of Twist1 rescues the inhibitory effect of miR-361-5p on glioma cell migration and invasion. Cells were co-transfected with the miR361-5p mimics and pcDNA/Twist1 vector. (A) Cell migration and (B) invasion of glioma cells were detected by Transwell assay; ${ }^{*}$ p $<0.05$ vs. the scrambled control; ${ }^{\&} \mathrm{p}<0.05$ vs. the miR-361-5p mimics + vector.

of glioma (25-27). Thus, identification of glioma-associated miRNAs as biomarkers for glioma diagnosis, prognosis and treatment is of great importance. In the present study, we found that miR-361-5p expression was decreased in glioma samples and cell lines. Functional experiments revealed that miR-361-5p functioned as a tumor suppressor that inhibited glioma cell migration, invasion and EMT. Further data indicated that miR-361-5p functioned by targeting Twist1 in the glioma cells. Taken together, our data suggest that miR-361-5p plays an important role in glioma progression and represents a novel therapeutic agent for glioma.

Increasing evidence indicates that miR-361-5p is a tumor-associated miRNA that is frequently dysregulated in many human cancer types. It has been reported that miR-361-5p exhibits a lower expression pattern in castration-resistant prostate cancer and overexpression of miR-361-5p inhibits the malignant progression of prostate cancer by targeting signal transducer and activator of transcription-6 (22). The expression of miR-361-5p was found to be significantly decreased in colorectal and gastric cancer tissues, and was negatively correlated with lung metastasis and prognosis (23). Overexpression of miR-361-5p significantly inhibited proliferation, migration and invasion of colorectal and gastric cancer cells by inhibiting staphylococcal nuclease domain containing-1 (23). In hepatocellular carcinoma, the promoter of miR-361-5p was found to be significantly hypermethylated leading to reduced expression of miR-361-5p and overexpression of miR-361-5p inhibited the proliferation and invasion of hepatocellular carcinoma cells through targeting chemokine (C-X-C Motif) receptor 6 (21). Kanitz et al reported that miR-361-5p targets and represses vascular endothelial growth factor $\mathrm{A}$ in human cutaneous squamous cell carcinoma (28). These findings support a tumor-suppressor role of miR-361-5p. In accordance with these findings, our results support miR-361-5p as a tumor suppressor. We demonstrated that miR-361-5p was significantly decreased in glioma tissues, particularly in advanced tumor stages. We also demonstrated that overexpression of miR-361-5p inhibited glioma cell migration, invasion and EMT. However, an oncogenic role of miR-361-5p was previ- ously observed in cervical cancer (29). Thus, the precise role of miR-361-5p requires further investigation.

To date, the targets of miR-361-5p remain unknown. In the present study, we examined bioinformatic analysis predicted targets to identify the potential target of miR-361-5p in glioma cells. Notably, we identified Twist1, an important inducer of EMT, as a direct target of miR-361-5p. Overexpression of Twist1 has frequently been observed in a variety of human cancers playing an important role in regulating cancer initiation, progression and metastasis (12). Increasing evidence suggests that Twistl functions as a critical regulator of EMT (13,15-17). Twist1 promotes EMT by affecting downstream genes such as E-cadherin, an epithelial marker (30). It has been reported that Twist1 activates the transcription of Bmi-1, and that Twist1 and Bmi-1 cooperate to inhibit E-cadherin expression by binding to its promoter (24). High expression of Twist1 has been detected in a majority of human glioma-derived cell lines and human glioma tissues (18). In glioma patients, high expression of Twist1 predicts poor survival for glioma patients (19). Twist1 overexpression was found to promote cell invasion while suppression of Twist1 was found to inhibit cell invasion in glioma cells $(18,20)$. Therefore, targeted therapy using Twist 1 may have promising and potential therapeutic value for glioma treatment.

miRNAs are important mechanisms of Twist 1 posttranscriptional modification. A growing body of evidence has revealed that a variety of miRNAs act as negative regulators of Twist1 (31). miR-33b is reported to inhibit breast cancer metastasis through targeting Twist1 (32). miR-548c regulates cell migration and invasion of ovarian cancer cells by suppressing Twist1 (33). Liu et al reported that miR-1271 suppressed pancreatic cancer cell migration, invasion and EMT through targeting Twist1 (34). A more recent study found that miR-186 inhibits prostate cancer cell growth, vasculogenic mimicry formation and EMT via targeting Twist1 (35). However, the miRNAs targeting Twist1 in glioma have not been well investigated. In the present study, we found that miR-361-5p directly targeted Twist1 in glioma cells to regulate migration, invasion and EMT. The decreased expression of miR-361-5p partially 
contributed to the increased expression of Twist1 in the glioma cells that promoted tumorigenesis and metastasis. Our findings add new insight into the related molecular mechanism of glioma pathogenesis.

In conclusion, the present study was the first to show that miR-361-5p was decreased in glioma tissues and cell lines. miR-361-5p exhibited a suppressive effect on glioma cell migration, invasion and EMT indicating an important role in glioma metastasis. More importantly, we elucidated that the underlying mechanism involved direct regulation of its target gene Twist1. Although detailed knowledge regarding the precise role and mechanism of miR-361-5p in regulating tumorigenesis and metastasis of glioma remains incomplete, we confirmed the role of miR-361-5p as a tumor suppressor in glioma. These findings suggest that miR-361-5p may serve as a novel potential therapeutic target for glioma.

\section{Acknowledgements}

The present study was supported by the Social Research and Development Program of Shaanxi Province (no. 2016SF-110).

\section{References}

1. Westphal M and Lamszus K: The neurobiology of gliomas: From cell biology to the development of therapeutic approaches. Nat Rev Neurosci 12: 495-508, 2011.

2. Siegel RL, Miller KD and Jemal A: Cancer statistics, 2016. CA Cancer J Clin 66: 7-30, 2016.

3. Furnari FB, Fenton T, Bachoo RM, Mukasa A, Stommel JM, Stegh A, Hahn WC, Ligon KL, Louis DN, Brennan C, et al: Malignant astrocytic glioma: Genetics, biology, and paths to treatment. Genes Dev 21: 2683-2710, 2007.

4. Pollack IF: Neuro-oncology: Therapeutic benefits of reirradiation for recurrent brain tumors. Nat Rev Neurol 6: 533-535, 2010.

5. Pang JC, Kwok WK, Chen Z and Ng HK: Oncogenic role of microRNAs in brain tumors. Acta Neuropathol 117: 599-611, 2009.

6. Silber J, James CD and Hodgson JG: microRNAs in gliomas: Small regulators of a big problem. Neuromolecular Med 11: 208-222, 2009.

7. Bartel DP: MicroRNAs: Genomics, biogenesis, mechanism, and function. Cell 116: 281-297, 2004.

8. Winter J, Jung S, Keller S, Gregory RI and Diederichs S: Many roads to maturity: microRNA biogenesis pathways and their regulation. Nat Cell Biol 11: 228-234, 2009.

9. Manikandan J, Aarthi JJ, Kumar SD and Pushparaj PN: Oncomirs: The potential role of non-coding microRNAs in understanding cancer. Bioinformation 2: 330-334, 2008.

10. Zhang Y, Dutta A and Abounader R: The role of microRNAs in glioma initiation and progression. Front Biosci 17: 700-712, 2012.

11. Besse A, Sana J, Fadrus P and Slaby O: MicroRNAs involved in chemo- and radioresistance of high-grade gliomas. Tumour Biol 34: 1969-1978, 2013.

12. Zhu QQ, Ma C, Wang Q, Song Y and Lv T: The role of TWIST1 in epithelial-mesenchymal transition and cancers. Tumour Biol 37: 185-197, 2016.

13. Kang Y and Massagué J: Epithelial-mesenchymal transitions: Twist in development and metastasis. Cell 118: 277-279, 2004.

14. Kalluri R and Weinberg RA: The basics of epithelial-mesenchymal transition. J Clin Invest 119: 1420-1428, 2009.

15. Tam WL and Weinberg RA: The epigenetics of epithelialmesenchymal plasticity in cancer. Nat Med 19: 1438-1449, 2013.

16. Taube JH, Herschkowitz JI, Komurov K, Zhou AY, Gupta S Yang J, Hartwell K, Onder TT, Gupta PB, Evans KW, et al: Core epithelial-to-mesenchymal transition interactome gene-expression signature is associated with claudin-low and metaplastic breast cancer subtypes. Proc Natl Acad Sci USA 107: $15449-15454,2010$.
17. Yang $\mathrm{MH}$ and Wu KJ: TWIST activation by hypoxia inducible factor-1 (HIF-1): Implications in metastasis and development. Cell Cycle 7: 2090-2096, 2008.

18. Elias MC, Tozer KR, Silber JR, Mikheeva S, Deng M, Morrison RS, Manning TC, Silbergeld DL, Glackin CA, Reh TA, et al: TWIST is expressed in human gliomas and promotes invasion. Neoplasia 7: 824-837, 2005.

19. Nordfors K, Haapasalo J, Mäkelä K, Granberg KJ, Nykter M, Korja M, Paavonen T, Haapasalo H and Soini Y: Twist predicts poor outcome of patients with astrocytic glioma. J Clin Pathol 68: 905-912, 2015.

20. Mikheeva SA, Mikheev AM, Petit A, Beyer R, Oxford RG, Khorasani L, Maxwell JP, Glackin CA, Wakimoto H, González-Herrero I, et al: TWIST1 promotes invasion through mesenchymal change in human glioblastoma. Mol Cancer 9: 194, 2010.

21. Sun JJ, Chen GY and Xie ZT: MicroRNA-361-5p inhibits cancer cell growth by targeting CXCR6 in hepatocellular carcinoma. Cell Physiol Biochem 38: 777-785, 2016.

22. Liu D, Tao T, Xu B, Chen S, Liu C, Zhang L, Lu K, Huang Y, Jiang L, Zhang X, et al: MiR-361-5p acts as a tumor suppressor in prostate cancer by targeting signal transducer and activator of transcription-6 (STAT6). Biochem Biophys Res Commun 445: 151-156, 2014.

23. Ma F, Song H, Guo B, Zhang Y, Zheng Y, Lin C, Wu Y, Guan G, Sha R, Zhou Q, et al: MiR-361-5p inhibits colorectal and gastric cancer growth and metastasis by targeting staphylococcal nuclease domain containing-1. Oncotarget 6: 17404-17416, 2015.

24. Yang MH, Hsu DS, Wang HW, Wang HJ, Lan HY, Yang WH, Huang $\mathrm{CH}$, Kao SY, Tzeng CH, Tai SK, et al: Bmil is essential in Twist1-induced epithelial-mesenchymal transition. Nat Cell Biol 12: 982-992, 2010.

25. Kwak PB, Iwasaki S and Tomari Y: The microRNA pathway and cancer. Cancer Sci 101: 2309-2315, 2010.

26. Karsy M, Arslan E and Moy F: Current progress on understanding microRNAs in glioblastoma multiforme. Genes Cancer 3: 3-15, 2012.

27. Tivnan A and McDonald KL: Current progress for the use of miRNAs in glioblastoma treatment. Mol Neurobiol 48: 757-768, 2013.

28. Kanitz A, Imig J, Dziunycz PJ, Primorac A, Galgano A, Hofbauer GF, Gerber AP and Detmar M: The expression levels of microRNA-361-5p and its target VEGFA are inversely correlated in human cutaneous squamous cell carcinoma. PLoS One 7: e49568, 2012.

29. Wu X, Xi X, Yan Q, Zhang Z, Cai B, Lu W and Wan X: MicroRNA-361-5p facilitates cervical cancer progression through mediation of epithelial-to-mesenchymal transition. Med Oncol 30: 751, 2013.

30. Sun T, Zhao N, Zhao XL, Gu Q, Zhang SW, Che N, Wang XH, Du J, Liu YX and Sun BC: Expression and functional significance of Twist1 in hepatocellular carcinoma: Its role in vasculogenic mimicry. Hepatology 51: 545-556, 2010.

31. Haga CL and Phinney DG: MicroRNAs in the imprinted DLK1-DIO3 region repress the epithelial-to-mesenchymal transition by targeting the TWIST1 protein signaling network. J Biol Chem 287: 42695-42707, 2012.

32. Lin Y, Liu AY, Fan C, Zheng H, Li Y, Zhang C, Wu S, Yu D, Huang Z, Liu F, et al: MicroRNA-33b inhibits breast cancer metastasis by targeting HMGA2, SALL4 and Twist1. Sci Rep 5: 9995, 2015

33. Sun X, Cui M, Zhang A, Tong L, Wang K, Li K, Wang X, Sun Z and Zhang H: MiR-548c impairs migration and invasion of endometrial and ovarian cancer cells via downregulation of Twist. J Exp Clin Cancer Res 35: 10, 2016.

34. Liu H, Wang H, Liu X and Yu T: miR-1271 inhibits migration, invasion and epithelial-mesenchymal transition by targeting ZEB1 and TWIST1 in pancreatic cancer cells. Biochem Biophys Res Commun 472: 346-352, 2016.

35. Zhao X, Wang Y, Deng R, Zhang H, Dou J, Yuan H, Hou G, Du Y, Chen Q and Yu J: miR186 suppresses prostate cancer progression by targeting Twist1. Oncotarget 7: 33136-33151, 2016. 